\title{
A Gain-Scheduling Model Predictive Controller for Blood Glucose Control in Type 1 Diabetes
}

\author{
Amjad Abu-Rmileh* and Winston Garcia-Gabin, Member, IEEE
}

\begin{abstract}
This paper presents a control strategy for blood glucose (BG) level regulation in type 1 diabetic patients. To design the controller, model-based predictive control scheme has been applied to a newly developed diabetic patient model. The controller is provided with a feedforward loop to improve meal compensation, a gain-scheduling scheme to account for different BG levels, and an asymmetric cost function to reduce hypoglycemic risk. A simulation environment that has been approved for testing of artificial pancreas control algorithms has been used to test the controller. The simulation results show a good controller performance in fasting conditions and meal disturbance rejection, and robustness against model-patient mismatch and errors in meal estimation.
\end{abstract}

Index Terms-Gain scheduling (GS), model predictive control (MPC), type 1 diabetes mellitus (T1DM).

\section{INTRODUCTION}

D IABETES mellitus is a disease characterized by pancreas inability to regulate blood glucose (BG) levels. For glucose to be transported into cells, insulin, which is a hormone produced by the pancreas, is needed. If the delivered insulin is less than required or no insulin is produced at all, BG concentrations elevate to abnormal levels. As a result, the human body loses its most important energy source, and the accumulated BG becomes toxic to several tissues and organs if not treated. This ineffective/insufficient or no insulin secretion is known as diabetes.

In type 1 diabetes (T1DM), the immune system attacks and destroys the insulin producing $\beta$-cells in the pancreas. Thus, for T1DM patients to maintain glucose level close to normal, they have to take exogenous insulin injections or infusion daily. Conventional therapy depends mainly on insulin injections, taken three or four times per day to replace the insulin function. If glucose level is not controlled carefully within a tight range, long-term complications, ketoacidosis, and hypoglycemic coma can occur, with the latter being more life-threatening. Beside the insulin problem, glucose counter-regulation system is severely impaired in T1DM; glucagon secretion in response to hypoglycemia is highly decreased with the increasing endogenous insulin deficiency.

Manuscript received June 9, 2009; revised August 10, 2009; accepted September 16, 2009. Date of publication October 20, 2009; date of current version September 15, 2010. This work was supported in part by the Spanish government under the Grants DPI-2007-66728-C02-01 and DPI-2007-66728C02-02, by the European Union through FEDER funds and by the Catalan government under Grant SGR-00296, and the BR research grant of the University of Girona for the first author. Asterisk indicates corresponding author.

${ }^{*}$ A. Abu-Rmileh is with the Department of Electrical, Electronics, and Control Engineering, University of Girona, 17071 Girona, Spain (e-mail: amjadhisham.ahmad@udg.edu).

W. Garcia-Gabin is with the Department of Electrical, Electronics, and Control Engineering, University of Girona, 17071 Girona, Spain (e-mail: winston.garcia@udg.edu).

Digital Object Identifier 10.1109/TBME.2009.2033663
Since 1970s, the idea of closed-loop artificial pancreas (AP) has been addressed as the solution to replace the existing treatment, and to improve the disease management (see [1] and [2]). While no commercial AP is currently available, the components of the AP, i.e., the continuous subcutaneous (s.c.) insulin infusion (CSII) pump, and the continuous glucose monitoring (CGM), are being used in open-loop setup. Although the sensors and pumps systems still have their limitations, their use has resulted in better clinical outcomes over conventional therapy [4]. A wide spectrum of control algorithms for closing the loop have been proposed (see reviews by Baquette et al. [2] and Takahashi et al. [3]). However, inter- and intrapatients variability in insulin sensitivity, variability in patient condition, mismatch between the models used to develop the control and real patients, as well as the limitations of the s.c. route used for glucose sensing and insulin delivery makes it very difficult to find a general and a reliable solution to the highly nonlinear problem of glycemic control and insulin therapy optimization.

This paper presents a model predictive control (MPC) algorithm for the BG control problem in T1DM, used with a newly developed glucose-insulin model. The proposed MPC scheme employs the following:

1) feedforward action, for the compensation of meal effect by anticipatory control action that prevents large hyperglycemic peaks;

2) hard constraints on insulin input, for patient's safety concerns and pump hardware specifications;

3) output constraints are implemented with unequal degrees of softness to penalize the hypoglycemic events more aggressively than hyperglycemic events, since hypoglycemia is more life threatening;

4) a gain-scheduling (GS) technique assigns specific insulin dosing profile for each glycemic range.

The designed controller has been evaluated in silico; a simulation environment with virtual diabetic subjects has been used to test and tune the controller.

This paper is structured as follows. Section II briefly describes the patient model and the simulation environment; the idea of the developed closed-loop algorithm, composed of MPC with feedforward control action and GS scheme, is given in Section III; the results obtained in the simulation scenarios are presented and discussed in Section IV; and finally, conclusions are drawn in Section V.

\section{Simulation Model}

Preclinical testing trial is a critical step to evaluate the performance and robustness of closed-loop control algorithms. Recently, in silico environments are being increasingly used for 
controllers testing [7], [14], and have shown ability to replace the animals' trials step in the development of clinical tools, while providing realistic results and covering a wider range of the variability observed among diabetic population [7]. These environments have as main building block a model that describes the T1DM patients (virtual subject). Many models have been investigated for in silico studies; these models range from simple linear models (e.g., Ackerman model) to nonlinear models (Bergman, and De Gaetano models) and comprehensive mathematical models (e.g., Cobelli, Sorensen, Hovorka, and Dalla Man models). For a detailed review on available models, see [15].

The meal model developed by Man et al. [8], [9] consists of a glucose and insulin compartments network linked by the control of glucose on insulin secretion, and by insulin on glucose utilization and endogenous production. The model has been modified to adapt for T1DM subjects and insulin exogenous infusion [10]. The model has been implemented in a simulation environment (UVa T1DM simulator) that has been accepted by the Food and Drug Administration (FDA) as a substitute for animals' trial in preclinical testing of closed-loop AP control algorithms [7]. In addition to the patient model, the simulator incorporates two other models: a sensor-related errors model to account for sensor noise and measurements' errors [7], [11], and a model for the s.c. insulin pump. In this paper, the controller has been tested with ten adults available in the training version of the simulator.

\section{CONTROller Design}

\section{A. Model Predictive Control (MPC)}

MPC is a control strategy that has considerably developed over the past few years. MPC is fundamentally based on discrete model of the system to be controlled. A model is used to predict the future system outputs, based on the past and current values, and on the proposed future control actions. These actions are calculated by optimizing a cost function where the future tracking error is considered and the system constraints, if any. MPC employs a receding horizon strategy-repeated displacement of the time horizon, while applying only the first control signal in the calculated sequence at each time step, with the rest of the sequence discarded.

MPC has many features that make it a competitive candidate for the BG control problem:

1) prediction property of MPC that allows for anticipatory and careful insulin delivery;

2) it can be useful to overcome the physiological delays associated with the s.c. route [18];

3) compensation for dead time, which exists in glucose problem;

4) feedforward control action to compensate for known sources of disturbance, such as meal intake;

5) constraints handling on system inputs and outputs.

MPC algorithms have been used in a number of AP publications, where the applicability of such controllers was demonstrated [19]-[23].
To apply a linear MPC, a good linearized version "approximation" of the nonlinear model proposed in [8] and [10] should be obtained. In this paper, the state-space approach has been chosen to formulate the MPC problem, since it can be easily extended to multivariable and nonlinear systems [17]. An average patient model (a patient whose parameters are the averages of the ten adults' parameters) has been obtained, and the nonlinear model is linearized at three different operating points $(65,120$, and $220 \mathrm{mg} / \mathrm{dL})$ in three glycemic ranges $(<70,70-180$, and $>180 \mathrm{mg} / \mathrm{dL}$ ), and the obtained models are descretized (with $T s=5 \mathrm{~min})$, and used to design three linear MPC's. The discrete linearized state-space models obtained from the nonlinear patient's model can be written as

$$
\begin{aligned}
x_{k+1} & =A x_{k}+B_{u} u_{k}+B_{v} v_{k}+E d_{k} \\
y_{k} & =C x_{k}+D_{v} v_{k}+D_{d} d_{k}
\end{aligned}
$$

where $A_{n \times n}, B_{n \times 1}, E_{n \times 1}, C_{1 \times n}$, and $D_{1 \times 1}$ are the matrices of the linearized model with $n$ being the system order $(n=13), x_{k}$ (deviation from linearization point) is the model states vector (13 states), $u_{k}$ (deviation from linearization point) is the insulin input in picomols per minute per kilogram, and converted to units per hour later in this paper for more convenient representation $(1 \mathrm{u}=6000 \mathrm{pmol}), v_{k}$ is the meal intake disturbance in milligrams, $d_{k}$ is the sensor errors in milligrams per deciliter, and $y_{k}$ is the measured glucose level in milligrams per deciliter (s.c. glucose plus sensor errors). The errors $d_{k}$ are modeled as integrated white noise in order to approximate the sensor errors presented in the UVa simulator (for errors model, see [11]).

The control action $u_{k}$ is governed by optimizing a quadratic cost function, penalizing predicted output deviations and control signal along some prediction horizons. The used cost function has the form

$$
\begin{aligned}
& \min _{\Delta u}\left\{\begin{array}{c}
J=\sum_{j=1}^{H_{p}}\left\|w^{y}(\hat{y}(k+j \mid k)-r(k+j))\right\|^{2} \\
+\sum_{j=1}^{H_{u}}\left\|w^{\Delta u} \Delta u(k+j \mid k)\right\|^{2}+q \varepsilon^{2}
\end{array}\right\} \\
& \text { s.t. } \\
& u_{\min } \leq u_{k} \leq u_{\max } \\
& \Delta u_{\min } \leq \Delta u_{k} \leq \Delta u_{\max } \\
& y_{\min }-\varepsilon V_{\min } \leq \hat{y}_{k} \leq y_{\max }+\varepsilon V_{\max }
\end{aligned}
$$

where $\hat{y}(k+j \mid k)$ is the $j$-step prediction of the output on data up to instant $k, r(k+j)$ is the reference glucose level, $\Delta u$ is the input increment, $H_{p}$ and $H_{u}$ are the prediction and control horizons, respectively, $w^{\Delta u}$ and $w^{y}$ are weights on the control action increments and the error between $y(k)$ and $r(k)$, respectively, $\varepsilon$ is a slack variable used for output constraints softening, $q$ is weight on the slack variable $\varepsilon, u_{\min / \max }, \Delta u_{\min / \max }$, and $y_{\min / \max }$ are the constraints imposed on the input, input increments, and output, respectively, and $V_{\min }$ and $V_{\max }$ are the relaxation variables.

Since hard output constraints may cause infeasibility in the optimization problem (e.g., because of unpredicted disturbances 
or model mismatch), $\varepsilon$ is defined for output constraints softening [16]. The weight $q$ on $\varepsilon$ penalizes the violation of the constraints. The larger the $q \varepsilon$ with respect to input and output weights, the more the constraint violation is penalized. The cost function in (2) is asymmetric; the lower and upper output constraints are subjected to unequal relaxation bands, and therefore, the constraints have different levels of softness. The unequal softness levels could be achieved by introducing the nonnegative relaxation variable $V_{\min }$ and $V_{\max }$, which represent the concern for relaxing the corresponding constraint; the larger the $\mathrm{V}$, the softer the constraint. The reason for such an asymmetry is to penalize the hypoglycemic events more aggressively than hyperglycemic events, since the former is more life-threatening. For the weights $w^{\Delta u}$ and $w^{y}$, the smaller $w$, the less important is the behavior of the corresponding variable on the overall performance index.

\section{B. GS Control (GSC)}

GSC is a well-known technique for controlling nonlinear systems by linear controllers. For a successful employment, GSC requires the identification of scheduling parameters that can be used to select the suitable linear controller for each operating range of the nonlinear system [26]. GSC overcomes the limitations of the basic linear approximation approach, which is valid only in a neighborhood of a single operating point. After identifying one or more scheduling parameters, the nonlinear model is linearized, and linear controllers for different operating ranges can be designed. Then, the set of linear controllers is implemented as a single controller whose parameters are changed by monitoring the scheduling variables. GSC schemes show a performance similar to that of nonlinear controllers applied to the same problems, with computational load being smaller in the GSC approach [27].

For the problem at hand, the GSC can be of vital importance for many reasons. In healthy subjects, the level of insulin activity varies between different glycemic ranges, being dominant in hyperglycemic range, in balance with glucagon action in normoglycemia and almost inactive in the hypoglycemic range where glucagon is dominant. Also, it is proved that the insulin therapy causes more degradation in the functionality of counterregulatory hormones that are working to correct the hypoglycemic episodes induced by insulin overdose [24], and consequently, results in higher possibility for hypoglycemic risk. Finally, considering the fact that hypoglycemia does not occur naturally in diabetic patients, but can result from insulin therapy [25], the control algorithm should avoid substituting one problem for another. Therefore, different weights should be imposed on the insulin dose depending on different BG ranges in order to deal with the iatrogenic hypoglycemia that is considered to be the main limiting factor in tight control of diabetes [24], [25].

A simplified diagram of the GS-MPC scheme is shown in Fig. 1. As can be seen in the figure, the scheduling variable used here is the output variable (i.e., measured glucose levels). Three different regions have been defined corresponding to the normo-, hyper-, and hypoglycemic ranges. The three linearized models obtained in Section III-A have been used to design the family of the MPC controllers. The GS is used to select between

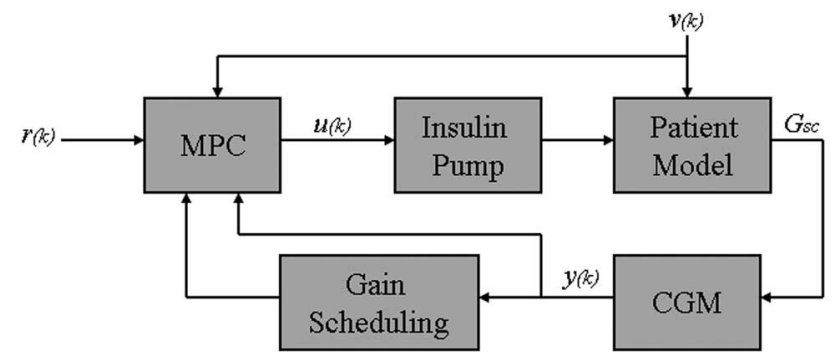

Fig. 1. MPC with GS, the CGM output is delivered to MPC as feedback loop, and to GS to select the controller to be used. Also, MPC receives the set-point value $r(k)$ and meal announcement signal $v(k)$ for feedforward action.

the three controllers depending on the measured glucose level. The scheduling thresholds are selected to be 80 and $160 \mathrm{mg} / \mathrm{dL}$, since the controller aims at maintaining normoglycemia, avoid reaching hypoglycemia, and reduce the elevated hyperglycemic peaks. The MPC receives the scheduling signal, the meal announcement, the reference glucose level $r(k)$, and the CGM readings. The pump receives the controller signal and deliver it as basal and/or bolus insulin.

\section{RESULTS}

The UVa simulator is equipped with many numerical and graphical metrics that can be used to evaluate the performance of the control algorithms. Among these, the primary metrics are: percentage of time within (70-180) $\mathrm{mg} / \mathrm{dL}$ range, low $\mathrm{BG}$ risk index (LBGI) or the hypoglycemic risk, and the "control variability grid analysis" (CVGA); for more details about these metrics and others, see [12] and [13].

\section{A. Scenario Setup}

Through the development of the controller, various scenarios have been simulated to study the main factors affecting the controller performance, including: the performance of feedforward control against meal disturbances (see Section IV-B1), controller ability to handle the erroneous estimation of carbohydrates' (CHO's) amount in meal (see Section IV-B2), superiority of GS-MPC over conventional MPC in dealing with interpatients variability (see Section IV-B3), and the benefit of controller's individualization for each patient (see Section IV-B4). Also, good set-point tracking and avoidance of overnight hypoglycemia have been taken into consideration in all scenarios.

Meal protocols that have been used in clinical and in silico trials are available in literature (see [6] and [10]). In our work, the simulation scenarios consider a three-day testing period, in which meal sizes and times are not restrictedly fixed, to account for patient real life where adherence to a fixed rule in meals' size and timing is difficult to achieve. The meals used in simulation are given in Table I.

The set point $r(k)$ for BG is selected to be $120 \mathrm{mg} / \mathrm{dL}$ since the glycemic target for T1DM patients at fasting conditions is about $100 \mathrm{mg} / \mathrm{dL}$ and the 2-h postprandial glucose level to be lower than $140 \mathrm{mg} / \mathrm{dL}$, and the constant mean value $120 \mathrm{mg} / \mathrm{dL}$ has been selected. The set point $120 \mathrm{mg} / \mathrm{dL}$ is frequently used 
TABLE I

DAILY CHOs AMOUNT

\begin{tabular}{|c|c|c|c|c|c|c|c|c|c|c|}
\hline \multirow{2}{*}{$\begin{array}{c}\text { Day } \\
\text { Meal size (gram } \mathrm{CHO})\end{array}$} & \multicolumn{3}{|c|}{ First } & \multicolumn{4}{|c|}{ Second } & \multicolumn{3}{|c|}{ Third } \\
\hline & 55 & 85 & 75 & 60 & 90 & 80 & 55 & 45 & 100 & 60 \\
\hline Total CHO daily (gram) & \multicolumn{3}{|c|}{215} & \multicolumn{4}{|c|}{290} & \multicolumn{3}{|c|}{205} \\
\hline
\end{tabular}

in clinical trials (see [5] and [6]). To start the in silico testing, the controller has been plugged into the simulator, so that the controller receives the signals, as in Fig. 1, and delivers the calculated insulin dose to the insulin pump.

During the tuning of the controller's parameters, the following have been considered, the prediction horizon $H_{p}$ is established since about $3 \mathrm{~h}$ are taken for the BG to return to steady state after a meal. A prediction horizon of $4 \mathrm{~h}$ is mainly used to consider the effect of insulin for $1 \mathrm{~h}$ more (i.e., to be more conservative in insulin dosing) and avoid hypoglycemic event that might result after a big meal. The 4-h (i.e., 48 sampling interval) horizon might result in a slightly higher hyperglycemic peaks, but it showed to be better than $3 \mathrm{~h}$ in dealing with hypoglycemic risk that is more life-threatening; for $H_{u}$, a length of 1 sampling interval ( $5 \mathrm{~min}$ ) is tested and considered be sufficient. For $y_{\min }$ and $y_{\max }$, the values have been selected to constrain the BG levels within the normoglycemic range (70$180 \mathrm{mg} / \mathrm{dL})$ and keep a safety margin, and therefore, the (110$160 \mathrm{mg} / \mathrm{dL}$ ) set has been used for most patients. The values for $V_{\min } / V_{\max }$ have been selected in such a manner to penalize hypoglycemia more aggressively than hyperglycemia, and avoid infeasibility in optimization that could result from hard constraints. The $V_{\min } / V_{\max }$ is a key tuning parameter that can be increased or decreased depending on patient's dynamics and sensitivity (e.g., smaller $V_{\min } / V_{\max }$ for patients with higher risk of hypoglycemia). The weights $w^{y}$ and $w^{\Delta u}$ have been selected such that the reference tracking (BG closed to set point) has a larger priority than the change in the insulin input during the optimization. Finally, for the input constraints $u_{\max }, u_{\min }$, and $\Delta u$, the values have been selected so that they remain within the maximum allowable values for patient's safety, and achieve the pump hardware specification and limitations. The controller parameters used in Section IV-B1-B3 are: $H_{p}=48, H_{u}=1$, $w^{y}=1, w^{\Delta u}=0.001, q=10^{5}, V_{\min } / V_{\max }=0.0001 / 0.01$, $y_{\min }=110 \mathrm{mg} / \mathrm{dL}, y_{\max }=160 \mathrm{mg} / \mathrm{dL}, u_{\min }=0 \mathrm{U} / \mathrm{h}, u_{\max }=$ $70 \mathrm{U} / \mathrm{h}$, and $|\Delta u|_{\max }=0.9 u_{\max }=63 \mathrm{U} / \mathrm{h}$. Some of these parameters (mainly $H_{p}, V_{\min } / V_{\max }$, and $y_{\min }$ and $y_{\max }$ ) have been subjected to further tuning in Section IV-B4 and B5 scenarios to achieve better individualized control for some patients.

In the scenarios 1-3 in Section IV-B, a general controller configuration has been used in the simulation; the models incorporated in the MPC are the three linearized models of an average patient (patient that has the average values of the ten adults parameters), and the controller tuning parameters have not been modified for each patient, but adjusted to achieve good performance for the whole group. Conversely, in the scenarios in 4 and 5 , while the same average models are used, individual controller tuning has been performed for each patient separately, depending on each patient's characteristics, ten tuning procedures have
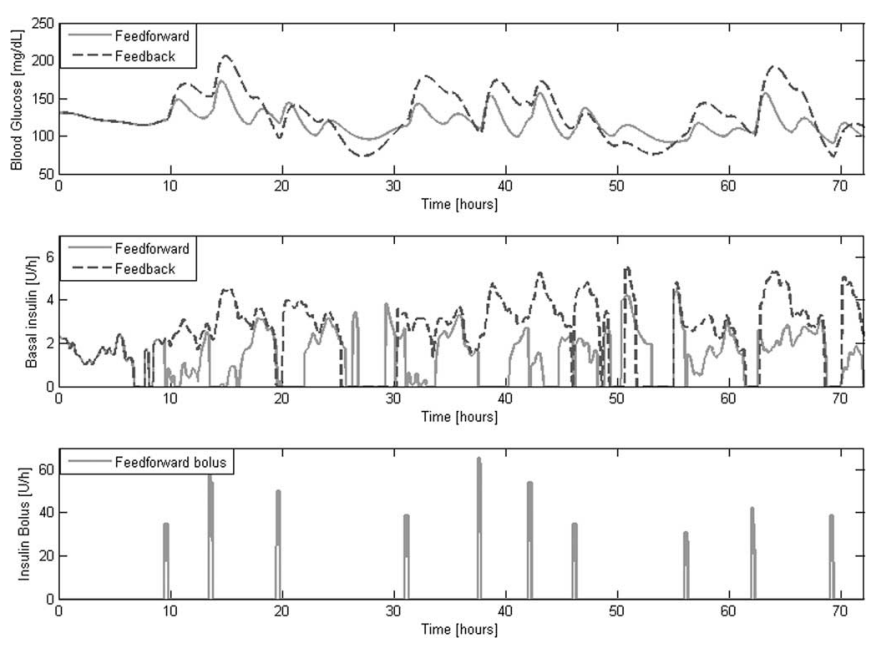

Fig. 2. (Top) Adult 5 BG trace with (solid) and without (dashed) feedforward loop. (Middle) Insulin infusion with (solid) and without (dashed) feedforward loop. (Bottom) Feedforward insulin bolus.

been performed for the ten adults to explore the possibility of further performance improvement.

\section{B. Discussion}

1) Feedforward Loop: To study the effectiveness of the feedforward action, two simulations have been performed with and without the feedforward loop. When feedforward loop is implemented, as in Fig. 1, the controller is announced about the upcoming meal, and the value of the meal is included in the prediction of future values of BG [see $v_{k}$ in (1)], and consequently, the meal effect on BG will be considered in calculating the future insulin dose.

While in the feedback-alone scenario, the $v_{k}$ value is always zero and the meal effect is not considered in the prediction, and therefore, the insulin infusion is depending on the CGM feedback signal and the MPC predictions. Thus, the meal correction only starts after the meal effect appears in the CGM readings.

Fig. 2 shows that the controller performs well in both configurations; however, it can be noticed that better results have been obtained with the feedforward loop: less fluctuations in BG levels (narrower range of BG readings), better set point tracking (mean BG is closer to $120 \mathrm{mg} / \mathrm{dL}$ ), and lower hyperglycemic peaks, since the feedforward starts to deliver insulin before the meals effect appears in the CGM feedback loop. Also, it should be noticed that the feedforward is used to activate the pump bolus feature, while in the feedback-alone scenario, the insulin infusion makes use of the pump basal only. Feedforward loop has been tested with the ten adults, and proved to give better results 


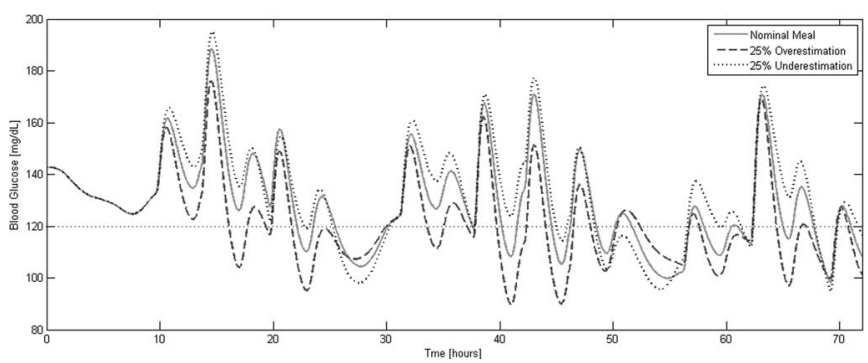

Fig. 3. Adult 5 BG trace with nominal meal (solid), meal $25 \%$ overestimation errors (dashed), and underestimation errors (dotted).

than feedback-alone configuration; therefore, feedforward has been implemented in all the following scenarios.

2) Meal Estimation Error: Whereas meal announcement (informing the controller about the upcoming meal) is essential for good feedforward compensation, it is not uncommon that the estimation for $\mathrm{CHO}$ content of the meal be incorrect, or that the calculated value is not totally delivered into blood stream. Therefore, a feedforward controller should have a good level of robustness against both meal over- and under estimation errors. The controller has been tested against over- and underestimation errors up to $25 \%$. Fig. 3 shows the glucose levels obtained for one patient (adult 5) with the $25 \%$ errors. For the three scenarios (nominal meal, and overestimation errors, and underestimation errors), the controller has been able to keep the BG level within the safe range of normoglycemia. It was surprising that for all patients, the controller response has not been affected by small and moderate estimation errors, while only minimum performance degradation has been noticed with $25 \%$ errors.

3) GS-MPC Versus MPC: To study the feasibility of the GS-MPC and its advantage over the conventional MPC, both controllers have been tested with the ten adults' population. While the MPC uses a single linear average model of the ten adults, the GS-MPC consists of three controllers, each of them contains one of the three average models that have been extracted in the hyper-, hypo-, and normoglycemic ranges. The GS is used to select between these three controllers in function of the glucose level provided by CGM. The CVGA tool developed in [12] is provided in the simulator as a tool to evaluate the control algorithm with the whole population. For the controller to be considered as "working good" or providing "optimal control," it should keep the patients within the A and B zones of the CVGA.

Fig. 4 shows the CVGA for the results obtained by the two controllers with ten adults during three days period (30 points, each represents the $\mathrm{BG} \mathrm{min} / \mathrm{max}$ pair obtained for one patient during one day). As the figure indicates, the GS-MPC has a superior performance over the conventional MPC, especially with those patients who exhibit higher nonlinear behavior and higher insulin sensitivity. It can be seen that by using the GS, the controller can handle each region separately and provide better control than the conventional MPC that controls the whole glycemic range with a single linear model. The MPC with GS is able to keep all the patients within the A and B zones. None of the patients is within the $\mathrm{D}$ and $\mathrm{F}$ dangerous zones.
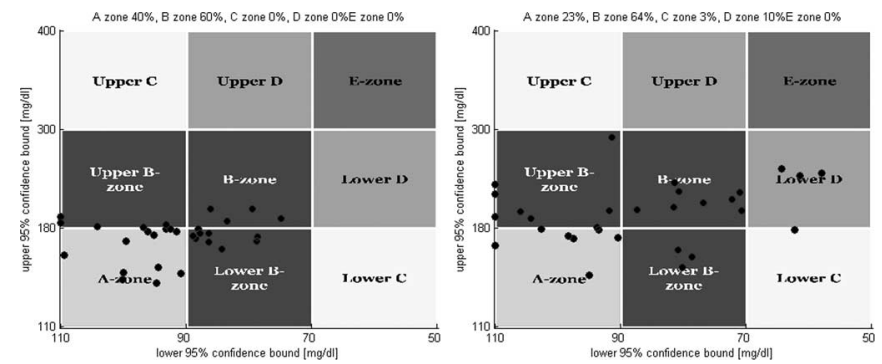

Fig. 4. CGVA for ten adults' population. (left) With GS-MPC. (Right) With conventional linear MPC. Each dot represents one subject during one day of the simulation period.

Numerical metrics available in the simulator, such as: percentage within $70-180 \mathrm{mg} / \mathrm{dL}$ range, percentage below $50 \mathrm{mg} / \mathrm{dL}$, percentage above $280 \mathrm{mg} / \mathrm{dL}$, LBGI and high BG risk index (HBGI), total risk index, and $\mathrm{BG}$ means, have been used to get a detailed idea about the GS-MPC controller performance. Table II demonstrates the obtained numerical results. It can be seen from the table that tight control has been achieved for all patients. None of the patient reaches the hypoglycemic range (below $70 \mathrm{mg} / \mathrm{dL}$ ), none of them reaches severe hyperglycemia (above $280 \mathrm{mg} / \mathrm{dL}$ ), and the risk indexes show that all patients are within the safe range of glycemia (e.g., the LBGI is below the 2.5 limits of "low-risk" range) [13], [28].

4) Individual Controller: In the previous scenarios, a general controller configuration has been tested, i.e., the controller incorporates the models of an "average patient" and the tuning parameters have not been modified between patients, and good performance has been verified. However, since a wide range of interpatients variability can be observed among diabetic population, it is obvious that a general configuration controller tuned for the whole population will not be as good as an individual controller that is tuned for each patient separately. To explore the possibility of further controller improvement, individual tuning (mainly for $H_{p}, V_{\min } / V_{\max }$, and $y_{\min }$ and $y_{\max }$ ) has been performed for each patient separately. The tuning procedure is performed by using patients' parameters that can be obtained in clinical tests, such as: the $\mathrm{CHO}-$ insulin ration $(\mathrm{CR})$ that gives the insulin units needed to eliminate the effect of certain amount of $\mathrm{CHO}$ grams, the correction factor $(\mathrm{CF})$ that represents the insulin units needed to drop the BG by a certain number of points (in milligrams per deciliter), the patients' fasting BG, and the patients' body weights (BWs). These parameters and others are available in the UVa simulator.

The obtained results in Table III emphasize the fact that the good performance in the previous scenario can be further improved by individual tuning. The population stays within the acceptable range of $70-180 \mathrm{mg} / \mathrm{dL}$ for $99.7 \%$ of the three-days period, the fluctuation in BG is minimized (narrower range between pre- and postmeal BG means), lower risk indexes have been achieved for all patients, and insulin overdosing problem has not occurred (no postprandial hypoglycemic events) despite of the realistic meals' values that have been used in the testing scenarios.

5) In Silico Validation: Although the good performance obtained in the previous sections indicates that the GS-MPC 
TABLE II

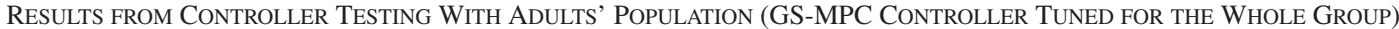

\begin{tabular}{|c|c|c|c|c|c|c|c|c|c|c|c|}
\hline Subject & mean $B G$ & $\begin{array}{c}\text { pre meal } \\
B G\end{array}$ & $\begin{array}{c}\text { post meal } \\
B G \\
\end{array}$ & $\begin{array}{c}\text { \% below } \\
\text { target }\end{array}$ & $\begin{array}{c}\text { \% above } \\
\text { target }\end{array}$ & $\begin{array}{l}\% \text { below } \\
50 \mathrm{mg} / \mathrm{dl}\end{array}$ & $\begin{array}{c}\text { \% above } \\
280 \mathrm{mg} / \mathrm{dl}\end{array}$ & $\begin{array}{c}\text { \% within } \\
\text { target }\end{array}$ & $L B G I$ & $H B G I$ & $\begin{array}{c}\text { risk } \\
\text { index }\end{array}$ \\
\hline Adult 1 & 123,71 & 114,18 & 145,35 & 0 & 1,23 & 0 & 0 & 98,77 & 0,37 & 1,01 & 1,38 \\
\hline Adult 2 & 121,44 & 118,68 & 132,22 & 0 & 0 & 0 & 0 & 100 & 0,05 & 0,47 & 0,52 \\
\hline Adult 3 & 130.5 & 135,07 & 138,62 & 0 & 1,15 & 0 & 0 & 98,85 & 0,12 & 1,55 & 1,67 \\
\hline Adult 4 & 132,4 & 127,12 & 157,7 & 0 & 3,9 & 0 & 0 & 96,1 & 0,15 & 1,83 & 1,98 \\
\hline Adult 5 & 129,1 & 126,44 & 158,54 & 0 & 2,2 & 0 & 0 & 97,8 & 0,06 & 1,46 & 1,52 \\
\hline Adult 6 & 128,72 & 122,38 & 155,6 & 0 & 1,58 & 0 & 0 & 98,42 & 0,05 & 1,09 & 1,14 \\
\hline Adult 7 & 126,69 & 117,9 & 149,48 & 0 & 1,93 & 0 & 0 & 98,07 & 0,17 & 1,23 & 1,4 \\
\hline Adult 8 & 118,13 & 117,2 & 125,49 & 0 & 0 & 0 & 0 & 100 & 0,11 & 0,32 & 0,43 \\
\hline Adult 9 & 131,09 & 128,27 & 139,04 & 0 & 1,65 & 0 & 0 & 98,35 & 0,22 & 1,6 & 1,82 \\
\hline Adult 10 & 133,66 & 128,55 & 161,38 & 0 & 2,4 & 0 & 0 & 97,6 & 0,18 & 1,99 & 2,17 \\
\hline Mean & 127,22 & 123,58 & 146,34 & 0 & 1,60 & 0 & 0 & 98,4 & 0,15 & 1,26 & 1,40 \\
\hline
\end{tabular}

TABLE III

Results From GS-MPC CONTROLler Testing With Adults’ Population, Individual Controller Tuning

\begin{tabular}{|c|c|c|c|c|c|c|c|c|c|c|c|}
\hline Subject & mean $B G$ & $\begin{array}{c}\text { pre meal } \\
B G\end{array}$ & $\begin{array}{c}\text { post meal } \\
B G\end{array}$ & $\begin{array}{c}\text { \% below } \\
\text { target }\end{array}$ & $\begin{array}{c}\text { \% above } \\
\text { target }\end{array}$ & $\begin{array}{l}\% \text { below } \\
50 \mathrm{mg} / \mathrm{dl}\end{array}$ & $\begin{array}{c}\text { \% above } \\
280 \mathrm{mg} / \mathrm{dl}\end{array}$ & $\begin{array}{c}\% \text { within } \\
\text { target }\end{array}$ & $L B G I$ & HBGI & $\begin{array}{c}\text { risk } \\
\text { index }\end{array}$ \\
\hline Adult 1 & 122,27 & 114,99 & 138,91 & 0 & 0 & 0 & 0 & 100 & 0,37 & 0,82 & 1,2 \\
\hline Adult 2 & 120,4 & 118,77 & 129,88 & 0 & 0 & 0 & 0 & 100 & 0,03 & 0,38 & 0,41 \\
\hline Adult 3 & 129,1 & 137,43 & 133,19 & 0 & 0 & 0 & 0 & 100 & 0,11 & 1,36 & 1,47 \\
\hline Adult 4 & 130,49 & 126,12 & 156,94 & 0 & 1,41 & 0 & 0 & 98,59 & 0,07 & 1,48 & 1,56 \\
\hline Adult 5 & 129,55 & 129,24 & 151,32 & 0 & 0 & 0 & 0 & 100 & 0,02 & 1,12 & 1,14 \\
\hline Adult 6 & 126,89 & 122,11 & 146,68 & 0 & 0 & 0 & 0 & 100 & 0,02 & 0,82 & 0,85 \\
\hline Adult 7 & 125,03 & 118,58 & 142,94 & 0 & 0,66 & 0 & 0 & 99,34 & 0,16 & 0,99 & 1,14 \\
\hline Adult 8 & 117,6 & 117,04 & 123,92 & 0 & 0 & 0 & 0 & 100 & 0,09 & 0,27 & 0,36 \\
\hline Adult 9 & 128,2 & 129,75 & 132,53 & 0 & 0,59 & 0 & 0 & 99,41 & 0,2 & 1,3 & 1,5 \\
\hline Adult 10 & 132,1 & 127,92 & 160,51 & 0 & 0 & 0 & 0 & 100 & 0,09 & 1,66 & 1,76 \\
\hline Mean & 126,16 & 124,20 & 141,68 & 0 & 0,27 & $\mathbf{0}$ & 0 & 99,73 & 0,12 & 1,02 & 1,14 \\
\hline
\end{tabular}

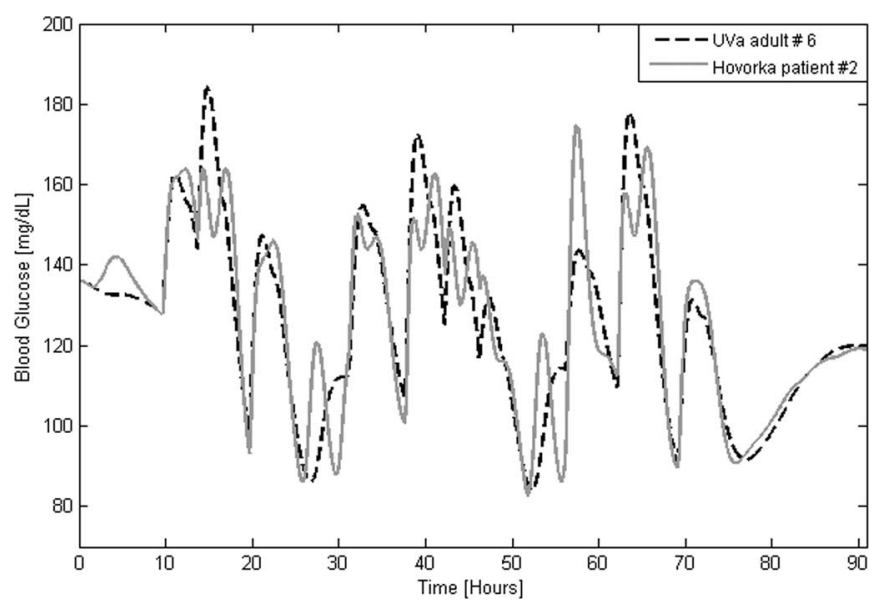

Fig. 5. Controller in silico validation: comparison between the performance of the designed controller with one of the UVa patients (adult 6) and one of the patients reported [21] (patient 2).

approach can nicely work and is applicable to the BG control problem, a validation could be done in silico by testing the controller performance against unknown dynamic model of the glucose-insulin system. For this purpose, the GS-MPC has been tested with the model developed by Hovorka et al. [21]. It is another nonlinear glucose-insulin model with a structure different from the one developed by Dalla Man and used in the UVa simulator.

Fig. 5 shows a comparison between two BG profiles obtained for one of the adults in the UVa simulator (adult 6), and one of the patients reported in [21] (patient 2). It can be seen that good control has been achieved for the hovorka patient although it is represented by a model that is totally different from the one implemented in the GS-MPC and used in the controller design. While satisfactory results could be achieved with the GS-MPC general configuration, the results in Fig. 5 have been obtained by considering the Hovorka patient as any other patient in the UVa simulator who needs individual tuning; therefore, the output constraints have been relaxed to $90 / 160 \mathrm{mg} / \mathrm{dL}$ for both patients. The figure also shows that two patients reach the $120 \mathrm{mg} / \mathrm{dL}$ value after the meals' disturbance is eliminated, even with the presence of the sensor errors. When applying the designed controller to another model, one should be careful about the specific units used for BG (e.g., in milligrams per deciliter or millimols per liter) and insulin input (e.g., in picomols per minute per kilogram or milliunits per minute) in each model.

The results shown in Tables II and III for general and individual tuning indicate that the total and premeal means of $\mathrm{BG}$ (not the sensor readings) are very close to the target value of $120 \mathrm{mg} / \mathrm{dL}$ in the presence of large sensor errors (e.g., about $30 \mathrm{mg} / \mathrm{dL}$ of deviation from real BG).

It should be mentioned that in all scenarios, the controller is initialized with an average basal insulin infusion required for the average patient (average of ten adults) to establish a $120 \mathrm{mg} / \mathrm{dL}$ fasting conditions, and since the set-point tracking has a larger priority on the insulin input, the initialization of GS-MPC with a value different from the one that is required for each patient will only be noticed in the first period of starting the control, because the MPC will establish a new basal depending on each patient.

Although the simulation studies demonstrate a good controller performance, additional points should be considered before moving the controller into clinical use where real conditions defer from those that can be generated in simulation environments. For instance, for night periods, where the risk of low BG 
readings increases, increased set-point value (e.g., $140 \mathrm{mg} / \mathrm{dL}$ ) can be used in attempt to reduce the insulin infusion, and consequently, the risk of hypoglycemia. Another action that can be considered is an alarm system that is activated when low BG level (e.g, $<80 \mathrm{mg} / \mathrm{dL}$ ) is detected. The alarm system can be used to give a warning sign for rapid intervention.

\section{CONCLUSION}

In this paper, a feedback-feedforward MPC controller with GS has been developed for BG closed-loop control problem. The applicability and the performance of the proposed control scheme have been evaluated in silico. The prediction ability of the MPC, enforced by effects of GSC and asymmetric cost function, resulted in a good controller performance and "adaptation" to different glucose ranges such that neither hypoglycemic events nor elevated hyperglycemia has been observed during simulation, although big-size meals have been used. A good level of robustness against meal over- and under estimation has been demonstrated. Also, the controller has shown good performance against interpatients variability and model-patient mismatch. In addition, while the GS-MPC has been driven by the CGM that can produce measurements errors up to $30 \mathrm{mg} / \mathrm{dL}$, the controller has been able to nicely reject the sensor errors effects. In order to validate the controller that has been developed, the model used in the controller design was not the same model that is used in the validation.

\section{REFERENCES}

[1] R. Hovorka, M. E. Wilinska, L. J. Chassin, and D. B. Dunger, "Roadmap to the artificial pancreas," Diabetes Res. Clin. Practice, vol. 74, pp. S178S182, 2006.

[2] B. W. Bequette, "A critical assessment of algorithms and challenges in the development of a closed-loop artificial pancreas," Diabetes Technol. Ther., vol. 7, no. 1, pp. 28-46, 2005.

[3] D. Takahashi, Y. Xiao, and F. Hu, "A survey of insulin dependent diabetes part II: Control methods," Int. J. Telemed. Appl, vol. 2008, doi: $10.1155 / 2008 / 739385$.

[4] D. Klonoff, "Continuous glucose monitoring. Roadmap for 21st century diabetes therapy," Diabetes Care, vol. 28, no. 5, pp. 1231-1239, 2005.

[5] G. M. Steil, A. E. Panteleon, and K. Rebrin, "Closed-loop insulin delivery - the path to physiological glucose control," Adv. Drug Del. Rev., vol. 56, no. 2, pp. 125-144, 2004.

[6] G. M. Steil, K. Rebrin, C. Darwin, F. Hariri, and M. F. Saad, "Feasibility of automating insulin delivery for the treatment of type 1 diabetes," Diabetes, vol. 55, no. 12, pp. 3344-3350, 2006.

[7] B. P. Kovatchev, M. Breton, C. D. Man, and C. Cobelli, "In silico preclinical trials: a proof of concept in closed-loop control of type 1 diabetes," Diabetes Sci. Technol., vol. 3, no. 1, pp. 44-55, 2009.

[8] C. D. Man, R. A. Rizza, and C. Cobelli, "Meal simulation model of the glucose-insulin system," IEEE Trans. Biomed. Eng., vol. 54, no. 10, pp. 1740-1749, Oct. 2007.

[9] C. D. Man, M. Camilleri, and C. Cobelli, "A system model of oral glucose absorption: Validation on gold standard data," IEEE Trans. Biomed. Eng., vol. 53 , no. 12 , pp. 2472-2478, Dec. 2006.

[10] C. D. Man, D. M. Raimondo, R. A. Rizza, and C. Cobelli, "GIM, simulation software of meal glucose-insulin model," Diabetes Sci. Technol., vol. 1, no. 3, pp. 323-330, 2007.

[11] M. Breton and B. P. Kovatchev, "Analysis, modeling, and simulation of the accuracy of continuous glucose sensors," Diabetes Sci. Technol., vol. 2, no. 5, pp. 1-10, 2008.

[12] L. Magni, D. M. Raimondo, C. D. Man, M. Breton, S. Patek, G. D. Nicolao, C. Cobelli, and B. P. Kovatchev, "Evaluating the efficacy of closed-loop glucose regulation via control variability grid analysis," Diabetes Sci. Technol., vol. 2, no. 4, pp. 630-635, 2008.
[13] B. P. Kovatchev, W. L. Clarke, M. Breton, K. Brayman, and A. McCall, "Quantifying temporal glucose variability in diabetes via continuous glucose monitoring: Mathematical methods and clinical application,” Diabetes Technol. Ther., vol. 7, no. 6, pp. 849-862, 2005.

[14] L. J. Chassin, M. E. Wilinska, and R. Hovorka, "Evaluation of glucose controllers in virtual environment: Methodology and sample application," Artif. Intell. Med., vol. 32, no. 3, pp. 171-181, 2004.

[15] F. Chee and T. Fernando, Closed-Loop Control of Blood Glucose. Berlin, Germany: Springer-Verlag, 2007.

[16] J. M. Maciejowski, Predictive Control With Constraints. London, U.K.: Prentice-Hall, 2002.

[17] E. F. Camacho and C. Bordons, Model Predictive Control, 2nd ed. London, U.K.: Springer-Verlag, 2004.

[18] R. Hovorka, "Continuous glucose monitoring and closed-loop systems," Diabetic Med., vol. 23, no. 1, pp. 1-12, 2006.

[19] R. S. Parker, F. J. Doyle, III, and NA. Peppas, "A model-based algorithm for blood glucose control in type I diabetic patients," IEEE Trans. Biomed. Eng., vol. 46, no. 2, pp. 148-157, Feb. 1999.

[20] S. Lynch and B. Bequette, "Model predictive control of blood glucose in type I diabetics using subcutaneous glucose measurements," in Proc. Amer. Control Conf., Anchorage, AK, 2002, vol. 5, pp. 4039-4043.

[21] R. Hovorka, V. Canonico, L. J. Chassin, U. Haueter, M. Massi-Benedetti, and M. Orsini, "Nonlinear model predictive control of glucose concentration in subjects with Type1 diabetes," Physiol. Meas., vol. 24, no. 4, pp. 905-920, 2004

[22] Z. Trajanoski and P. Wach, "Neural predictive controller for insulin delivery using the subcutaneous route," IEEE Trans Biomed. Eng., vol. 45, no. 9, pp. 1122-1234, 1998.

[23] L. Magni, D. M. Raimondo, L. Bossi, C. D. Man, G. D. Nicolao, B. P. Kovatchev, and C. Cobelli, "Model predictive control of type 1 diabetes: An in silico trial,"," Diabetes Sci. Technol., vol. 1, no. 6, pp. 804$812,2007$.

[24] V. J. Briscoe and S. N. Davis, "Hypoglycemia in Type 1 and Type 2 diabetes: Physiology, pathophysiology, and management," Clin. Diabetes, vol. 24, no. 3, pp. 115-121, 2006.

[25] D. LeRoith, S. I. Taylor, and J. M. Olefsky, Diabetes Mellitus: A Fundamental and Clinical Text. Baltimore, MD: Lippincott Williams \& Wilkins, 2004.

[26] W. J. Rugh and J. S. Shamma, "Research on gain scheduling," Automatica, vol. 36, no. 10, pp. 1401-1425, 2000.

[27] L. Chisci, P. Falugi, and G. Zappa, "Gain-scheduling MPC of nonlinear systems," Int. J. Robust Nonlinear Control, vol. 13, no. 3, pp. 295-230, 2003.

[28] B. P. Kovatchev, E. Otto, D. J. Cox, L. A. Gonder-Frederick, and W. L. Clarke, "Evaluation of a new measure of blood glucose variability in diabetes," Diabetes Care, vol. 29, no. 11, pp. 2433-2438, 2006.

Amjad Abu-Rmileh was born in Jerusalem, Israel, in 1984. He received the B.Sc. degree in biomedical engineering from Jordan University of Science and Technology, Ar Ramtha, Jordan, in 2006, and the M.Sc. degree in automation and system engineering from the University of Seville, Seville, Spain, in 2008. $\mathrm{He}$ is currently working toward his Ph.D. degree in automatics and electronic engineering at the University of Girona, Girona, Spain.

His current research interests include control in biomedical and biological systems, biomedical transport phenomena, and medical instrumentation.

Winston Garcia-Gabin (M'89) was born in Barquisimeto, Venezuela, in 1969. $\mathrm{He}$ received the Electrical Engineering degree and the Master of Science in Automation degree from University of the Andes, Merida, Venezuela, in 1994 and 1998, respectively, and the Ph.D. degree from the University of Seville, Seville, Spain, in 2002.

$\mathrm{He}$ is currently with the University of Girona, Girona, Spain, where he is a teacher and researcher. He was a process control engineer with several industries, and was engaged in the consultancy work. His current research interests include nonlinear control, modeling and instrumentation with applications in industrial process, and biosystems. 